Paper

\title{
Low latency information transfer based on precision time synchronization via wireless interferometry
}

\author{
Daijiro Koyama ${ }^{1}$, Yunzhuo Wang ${ }^{1}$, Nobuyasu Shiga ${ }^{2}$, \\ Satoshi Yasuda ${ }^{2}$, Nicolas Chauvet ${ }^{1,3}$, and Makoto Naruse ${ }^{1,3 a)}$ \\ ${ }^{1}$ Department of Mathematical Engineering and Information Physics, Faculty of \\ Engineering, The University of Tokyo, Bunkyo-ku, Tokyo 113-8656, Japan \\ ${ }^{2}$ Space-Time Standards Laboratory, Applied Electromagnetic Research Institute, \\ National Institute of Information and Communication Technology, Koganei, \\ Tokyo 184-8795, Japan \\ ${ }^{3}$ Department of Information Physics and Computing, Graduate School of \\ Information Science and Technology, The University of Tokyo, Bunkyo-ku, \\ Tokyo 113-8656, Japan \\ a) makoto_naruse@ipc.i.u-tokyo.ac.jp
}

Received July 27, 2020; Revised December 23, 2020; Published April 1, 2021

\begin{abstract}
The growing demand of high-bandwidth and low-latency information transfer in information and communication technologies such as data centers and in-vehicle networks has increased the importance of optical communication networks in recent years. However, complicated arbitration schemes can impose significant overheads in data transfer, which may inhibit the full exploitation of the potential of optical interconnects. Herein, we propose an arbitration protocol based on precision time synchronization via wireless two-way interferometry (Wi-Wi), and numerically validate its efficiency including the ability to impose a strict upper bound on the latency of data transfer. We introduce the notion of arbitration point (AP) for a designated time duration, which is shared by all nodes thanks to the time synchronization by Wi-Wi, to determine data transmission while ensuring avoiding collision. Compared with the conventional carrier sense multiple access/collision detection (CSMA/CD)-based approach, a significant improvement in the data transfer was observed especially in the cases with high traffic flow rate. Furthermore, we conducted a proof-of-principle experiment for Wi-Wi-based data transfer between two electrically connected nodes and confirmed that the skew was less than $300 \mathrm{~ns}$ and remained stable over time. Conversely, non-Wi-Wi-based data transfer exhibited huge and unstable skew. These results indicate that precision time synchronization is a promising resource to reduce the communication overheads and ensure low latency for future networks and real-time applications.
\end{abstract}

Key Words: Precision timing synchronization, Wireless two-way interferometry, Low latency information transfer 


\section{Introduction}

With the growing importance of high-bandwidth and low-latency information transfer, optical communication technologies are expected to play a crucial role in short-distance communication networks such as data centers and in-vehicle networks in addition to conventional long-distance applications [1]. For example, self-driving cars need a variety of sensors such as light detection and ranging (LIDAR) sensor and stereo cameras as well as their controllers and associated computation modules, which generate a large amount of data. Hence, high-speed and low-latency communication is required in these cars [2]. In particular, the reduction of communication delay or latency is crucial for devices requiring strict real-time operation.

To realize the full potential of optical data transmission, the number of optical-to-electrical (OE) and electrical-to-optical (EO) conversions should be reduced as much as possible; otherwise, they can increase the delay and energy requirements. Further, arbitration is an important issue that requires careful attention, and our study focuses on this aspect of data transfer. To facilitate the removal of $\mathrm{OE} / \mathrm{EO}$ conversions and to avoid unnecessary complexity, we have considered the case in which multiple nodes are connected in a unidirectional ring network. Since multiple nodes share access to a single bus, certain arbitration of communication is necessary to avoid collisions. Complex arbitration methods may impose significant overhead or delay, which can hinder the inherent advantages of optical signal transmission.

Meanwhile, Shiga et al. developed a precision time synchronization technique called wireless two-way interferometry, also known as Wi-Wi [3-5]. Wi-Wi realizes time synchronization between spatially separate positions by exchanging the time information via wireless communications: the principle is reviewed in Sec. 2. The principle and the technology of Wi-Wi were presented in [3], and their application to horizontal atmospheric delay measurement and distance variation monitoring has been successfully demonstrated in [4] and [5], respectively. In this study, we have employed such a time synchronization technique to facilitate arbitration of communication in optical networks. Whereas former works exploited the attribute of precision time synchronization by Wi-Wi to monitor physical variations $[4,5]$, here we utilize such property for information transfer, which is the main contribution of the present study.

In particular, we have proposed a simple arbitration method for a ring network in which all the nodes are time synchronized via Wi-Wi, and demonstrate its efficiency including the ability to impose a strict upper bound on the latency of data transfer. Here we introduce the notion of arbitration point (AP) to indicate a periodically shared designated time duration to determine data transmission while ensuring avoiding collision. What is important is that the timing of AP is commonly shared by all nodes thanks to the time synchronization provided by Wi-Wi. Compared with the conventional carrier sense multiple access/collision detection (CSMA/CD) approach, a significant improvement was observed in the proposed approach especially for the cases in which the traffic rate was high. Furthermore, we experimentally demonstrate the Wi-Wi-based data transfer between two electrically connected nodes and confirm the reduction in skew and its stability over time. We also show that the data transfer exhibits huge and unstable skew when Wi-Wi-based synchronization is turned off. These results pave the way for the realization of efficient and real-time communication by precise time synchronization for future applications.

\section{Principles}

\subsection{Basic principle of time synchronization via $\mathrm{Wi}-\mathrm{Wi}$}

Here we describe the basic principle of the time synchronization via Wi-Wi [3]. Wi-Wi employs socalled two-way time transfer (TWTT) technique [6], which is schematically illustrated in Fig. 1. The time at nodes $\mathrm{A}$ and $\mathrm{B}$ are denoted by $T_{\mathrm{A}}$ and $T_{\mathrm{B}}$, respectively, and the propagation delay between these sites is $\Delta$. To derive the time difference $\left(t_{c}=T_{\mathrm{B}}-T_{\mathrm{A}}\right)$ and the propagation delay between the two sites, each site sends its time information via the same physical route. Then, the time difference at $\mathrm{A}$ when the information of $T_{\mathrm{B}}$ is delivered is given by $\Delta_{\mathrm{atA}}=T_{\mathrm{A}}+\Delta-T_{\mathrm{B}}=-t_{c}+\Delta$, while that at $\mathrm{B}$ is $\Delta_{\mathrm{atB}}=t_{c}+\Delta$. Again, by exchanging these two time differences between each other, the time 
difference and propagation delay can be obtained as

$$
\begin{aligned}
& t_{c}=\left(\Delta_{\mathrm{atB}}-\Delta_{\mathrm{atA}}\right) / 2, \\
& \Delta=\left(\Delta_{\mathrm{atB}}+\Delta_{\mathrm{atA}}\right) / 2 .
\end{aligned}
$$

This allows us to synchronize the absolute time between the two sites.

TWTT was originally used for two-way satellite time and frequency transfer (TWSTFT), which is a time synchronization method based on geostationary satellites that is utilized in calibration of standard time $[6,7]$. TWSTFT achieves precise time synchronization with an accuracy of subpicosecond by carrier-phase two-way satellite frequency transfer (TWCP) [7], which is a technique based on carrier phase to derive precise time difference.

Wi-Wi is an extension of these techniques in which satellite communication is replaced with wireless communication, as represented by dashed arrows in Fig. 1, to achieve precision time synchronization. In our study, we employ modules that use $920-\mathrm{MHz}$ wireless communication to achieve a time synchronization with a standard deviation of $20 \mathrm{ps}$ [4].

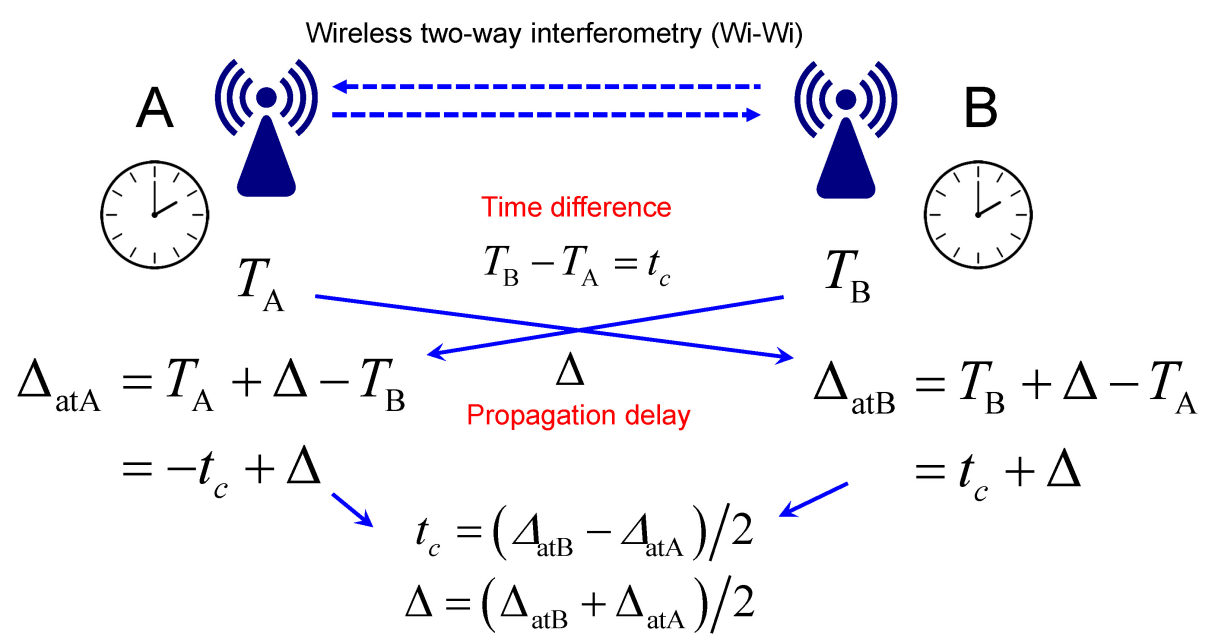

Fig. 1. Schematic diagram of wireless two-way interferometry (Wi-Wi). The two nodes, denoted by $\mathrm{A}$ and $\mathrm{B}$, exchange their time between each other, which allows them to realize the relative time differences denoted by $\Delta_{\text {atA }}$ and $\Delta_{\text {atB }}$ at nodes $\mathrm{A}$ and $\mathrm{B}$, respectively. These time differences are exchanged between each other, yields the relative time difference and the propagation delay denoted by $t_{c}$ and $\Delta$, respectively. Finally, the time at node $\mathrm{A}$ and $\mathrm{B}$ is synchronized using $t_{c}$.

\subsection{Timing-based arbitration in ring network}

We consider an optical network in which multiple computing nodes are connected in a unidirectional ring structure and all the nodes are time synchronized via Wi-Wi, as shown in Fig. 2(a). It is assumed that add/drop of information between the nodes and the ring is technically achievable at each node. Although the time synchronization via W-Wi of nodes arranged in ring structures has not been studied elsewhere, the ring topology of optical network itself has been well examined in the literature such as $[8]$ and $[9]$.

To describe the arbitration method based on timing, we introduce the notion of timing referred to as arbitration point (AP). AP is temporally transmitted in the ring regularly, as schematically illustrated by red-colored squares in Figs. 2(a) and 2(b). Thanks to the time synchronization of all the nodes, each node can independently recognize AP. The duration of an AP is defined by " 1 word".

Here we consider two states at AP: one is a 1-word-length non-zero-level signal defined by "1", while the other is a 1-word-length zero-level signal defined by "0". Each node can start sending the information content immediately after the time specified by AP. Here, we design the duration of the information content in such a way that the sum of total temporal length of AP and information 
(a)

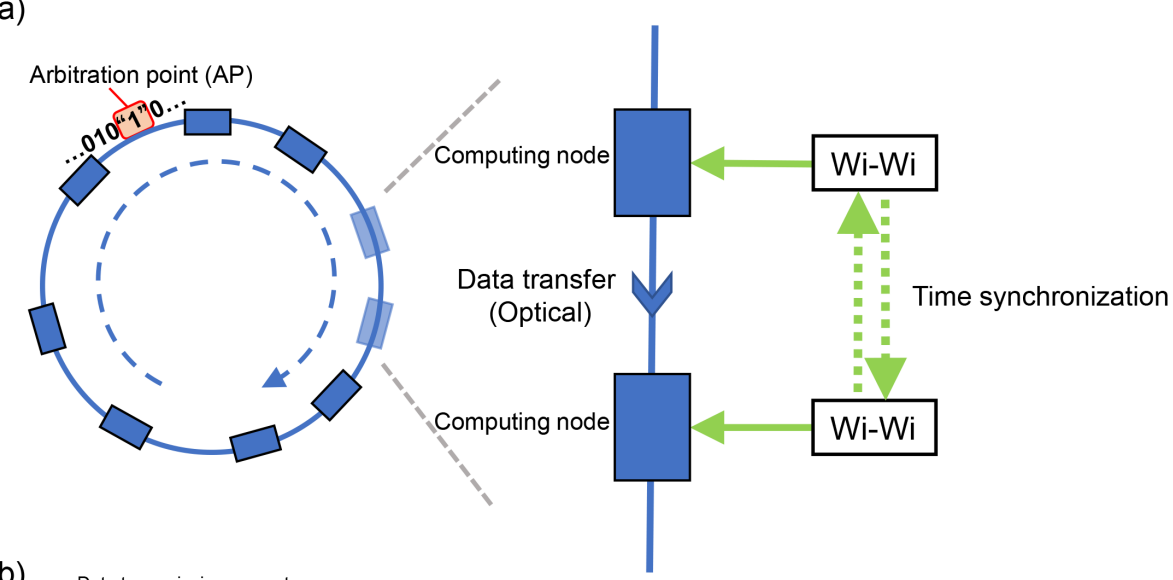

(b)

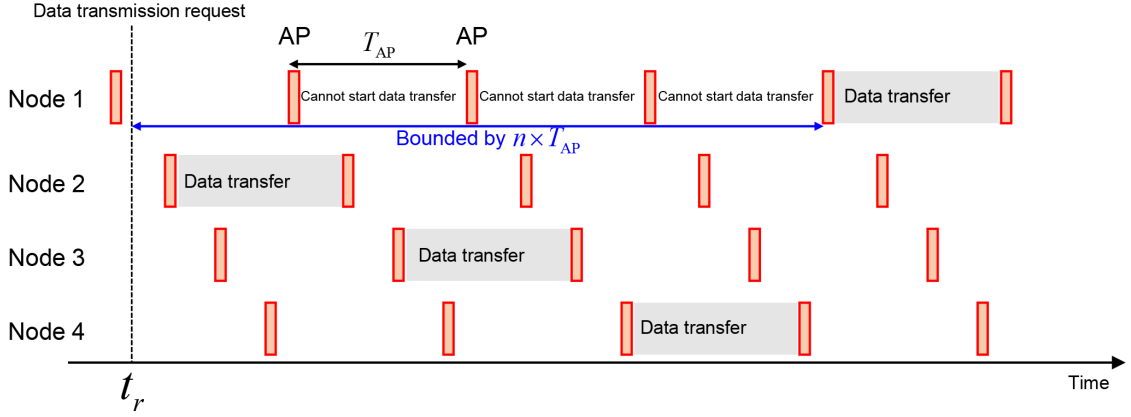

Fig. 2. (a) Schematic diagram of the system under study. Multiple computing nodes are connected in a unidirectional optical ring network while all nodes are synchronized via Wi-Wi. (b) Schematic illustration of the arbitration principle in the case when the number of nodes is four.

content corresponds to the physical length of the ring. This implies that no more than two information contents can exist in the ring.

In addition, to ensure maximum latency among any given nodes, a node does not send information contents consecutively by utilizing two consecutive APs. This rule also ensures that all the nodes have an equal chance of data transmission. With these constraints, when the number of nodes in a ring is $n$ and the AP interval is $T_{\mathrm{AP}}$, the transmission node can surely have the opportunity of data transmission no later than $n \times T_{\mathrm{AP}}$. In other words, we can pose a strict upper bound on the latency of data transfer. The flow of the proposed arbitration scheme is summarized in Algorithm 1. Detailed performance analysis will be demonstrated in Sec. 3 .

In the schematic illustration in Fig. 2(b), where the number of nodes is four, we assume that data transmission requests occur in all four nodes at the time $t_{r}$ indicated by the dashed line. As described above, each node experiences AP every $T_{\mathrm{AP}}$ cycle or gets potentially available data transmission opportunity. In the example in Fig. 2(b), among the four nodes, first Node 2 obtains the opportunity of data transmission, as indicated by a gray rectangle, since Node 2 is the first node which finds an AP while the ring is vacant. Subsequently, since Node 2 cannot transmit data after two consecutive APs, Node 3 gets the data transmission opportunity, followed by Node 4. Finally, Node 1 obtains the chance of data transmission. What is remarkable is that the time for every node to get the data transmission opportunity is bounded by $n \times T_{\mathrm{AP}}$ where $n$ is equal to four in Fig. 2(b).

It should be emphasized that such a simplified arbitration does not work without precision time synchronization among all nodes because the proposed protocol totally relies on the fact that all nodes share the timing of AP to conduct data transfer and avoid collisions. Conventionally, we cannot assume such time synchronization between nodes; hence, a complex arbitration protocol must be introduced. As a result, when we use the CSMA/CD scheme for example, the data transfer latency cannot be guaranteed before data transmission. Indeed, as we observed shortly in Sec. 3, the latency sometimes results in a radically large value, especially when the traffic rate is high. As discussed in Sec. 1, we consider that securing latency is one of the most important aspects in future networks, 
Algorithm 1 Timing-based arbitration in a ring network

1. Each node determines the timing of AP based on the precision timing synchronization via WiWi.

2. If a node wants to send information, i.e., the transmission queue contains certain message,

(a) The node transmits "1" at the timing of AP.

(b) Simultaneously, if the node receives "1" at the timing of AP from the ring, the node does not start transmitting information content to avoid a conflict. In addition, the node starts receiving the information content from the ring.

(c) When the node detects "0" at the timing of AP from the ring, the node starts transmitting the information content. Further, after finishing the transmission, "0" must be set in the next AP, i.e., the node cannot conduct consecutive data transmission.

3. If the node does not contain any information to be transmitted, i.e., the transmission queue is empty,

(a) The node transmits "0" at the timing of AP.

(b) Simultaneously, if the node receives "1" at the timing of AP from the ring, the node starts receiving the information content from the ring.

4. Return to Step 1.

including in-vehicle optical networks where the delay of certain sensory data transfer could lead to unexpected outcomes.

Finally, before proceeding to concrete numerical performance analysis, we have to emphasize the meaning of the CSMA/CD strategy, which is employed as a counterpart of the proposed method. Traditionally, CSMA/CD is known to be a media access control (MAC) method utilized in early Ethernet technology for local area networking, assuming a bus topology. In principle, there is no restriction regarding the length of packets, for example. Meanwhile, certain arbitration is indispensable in the unidirectional ring network shown in Fig. 2 to manage multiple accesses from multiple nodes while avoiding collisions. Furthermore, in the Wi-Wi-based proposed arbitration, certain conditions should be satisfied with respect to the length of a packet, not just the precision time synchronization among nodes.

Concerning all these conditions while considering a fair performance comparison, the term "CSMA/CD" in the present study does not literally mean the traditional CSMA/CD as a MAC method; it indicates a CSMA/CD-based arbitration strategy by only adapting the primal features of CSMA/CD to be matched with the ring network to be compared. For example, the packet size is set to be equivalent between CSMA/CD and the proposed method. The nature of light transmission in the ring is appropriately modeled in the CSMA/CD-based method. From these design principles and constraints, we consider that the CSMA/CD-based method adapted in the present study is a fair counterpart to be compared with the proposed Wi-Wi-based arbitration method. The details of the CSMA/CD-based arbitration method is described in Sec. 3.2.

\section{Performance analysis}

\subsection{Latency analysis of timing-based arbitration}

In order to verify the principle and to conduct performance analysis of the arbitration principles described in Sec. 2, we conducted numerical simulations to compare the latency or delay obtained 
by a conventional CSMA/CD-based approach and the proposed timing synchronization method. We evaluated two kinds of wait time. The first one is the difference between the time when a message enters the transmission queue and the time of its receipt by the receiver, which is called "Wait time 1". The second one is the difference between the arrival time of a message at the exit of the transmission queue and the time of its receipt by the receiver, which is called "Wait time 2".

Figures 3(a) and 3(b) show the Wait time 1 of CSMA/CD-based and the proposed method as a function of traffic rate, respectively. The details regarding the definition of the traffic rate are described in Sec. 3.2. Each symbol in these figures represents the average of the lower $x \%(x=10,20, \cdots, 90)$ of Wait time 1 over all transmitted messages as a function of the traffic rate that ranges from 0.01 to 0.99. In addition, the minimum and the maximum of Wait time 1 are also presented. Figure 3(a) shows that when the traffic rate is more than 0.2 in CSMA/CD, the wait time increases drastically. When the traffic rate is less than 0.2 , most of the messages are transmitted almost instantaneously, but some of messages wait for more than several tens of messages. On the other hand, as shown in Fig. 3(b), in the proposed method, even if the traffic rate is nearly 0.8 , nearly $90 \%$ of the messages are transmitted within 10 messages, indicating that the proposed method maintains good delay tolerance even when the traffic rate is high.

Figures 3(c) and 3(d) summarize the distributions of Wait time 2 obtained by CSMA/CD and the proposed method, respectively, based on the analysis of 1,500 messages for each traffic rate. Occasionally, the Wait time 2 can be unexpectedly long in CSMA/CD, while it is strictly bounded by maximum 1050 words in the proposed method. Further, it is evident from Fig. 3(d) that almost all the messages are sent within 8 or 9 cycles, and very few messages wait for 8 or 9 cycles in the proposed method even when the traffic rate is 0.9 or higher.

This remarkable reduction of Wait time 1 by the proposed method stems from its principle that allows all nodes to transmit data no later than $n \times T_{\mathrm{AP}}$ steps once it sits at the exit of the transmission queue as described in Section 2.2 and Fig. 2(b), unlike CSMA/CD-based arbitration where the waiting time cannot be bounded. Figure 4(a) shows the average of Wait time 2 of all the messages for each traffic rate. On average, the Wait time 2 of the proposed method is shorter than that of CSMA/CD
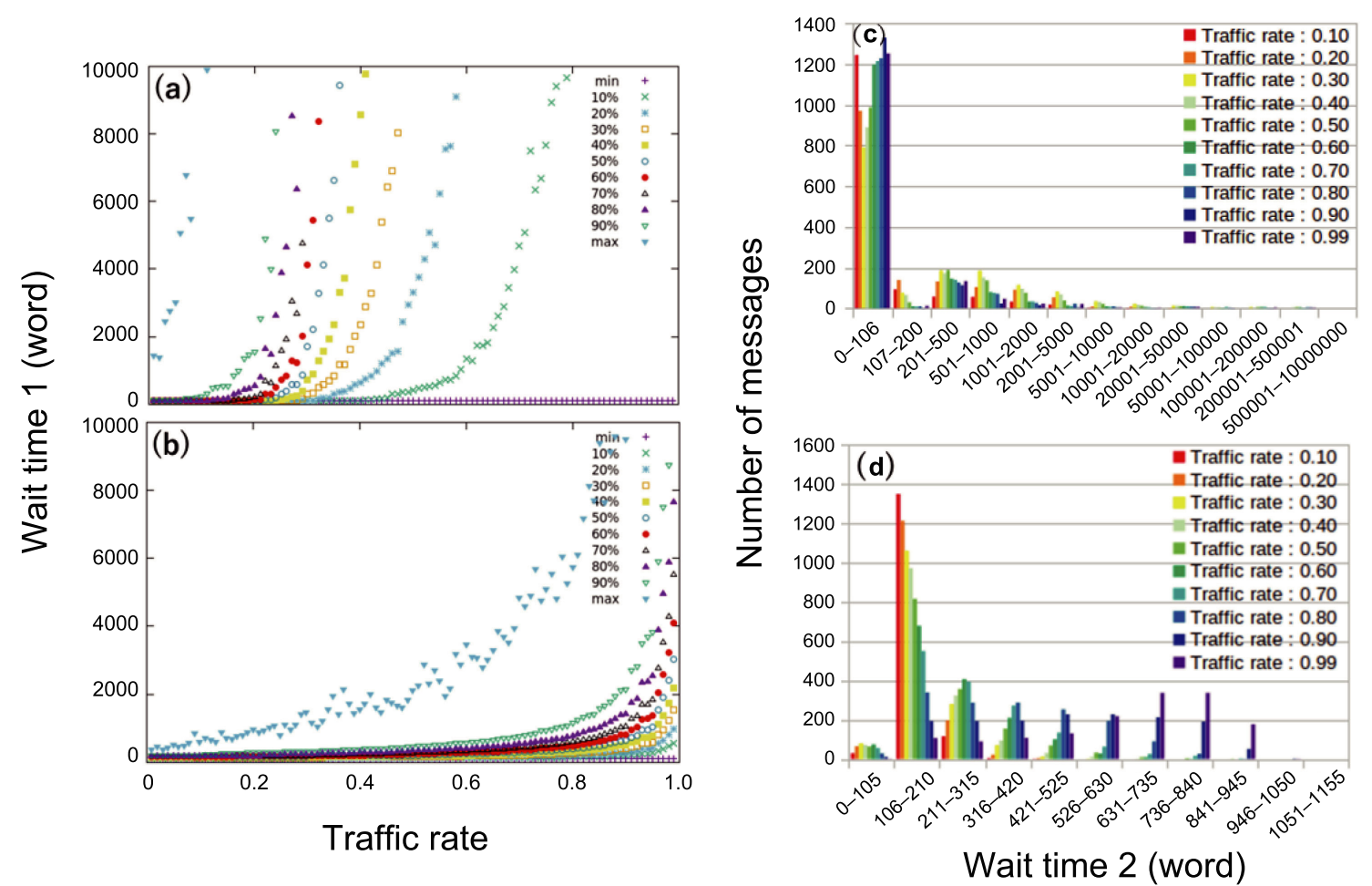

Fig. 3. Latency analysis by the proposed method. (a, b) Wait time 1 obtained by CSMA/CD-based (a) and proposed method (b). (c, d) Wait time 2 obtained by CSMA/CD-based (c) and proposed method (d). 


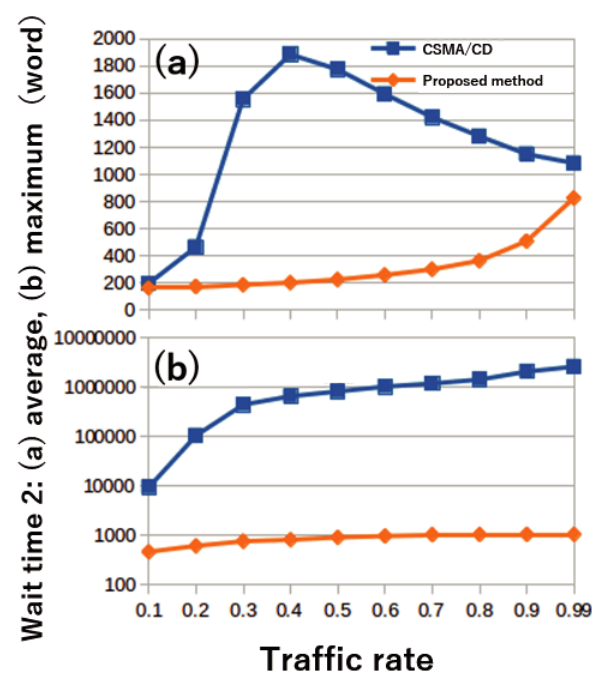

Fig. 4. Comparison of (a) the average and (b) the maximum value of Wait time 2 obtained by CSMA/CD-based and the proposed method.

regardless of the traffic rates. Meanwhile, even if the traffic rate is high, we observe that the average wait time in CSMA/CD is not very long. However, this is actually based on the fact that some of the messages in a CSMA/CD scheme are never transmitted during the simulation time, which are not taken into account in calculating the average value. That is, few messages suffer from extremely long wait times, which is also observed in Fig. 3(a). Indeed, as shown in Fig. 4(b), the maximum wait time in CSMA/CD exponentially increases with the increase in the traffic rate, which severely impacts the scenarios in which real-time operation or short latency is crucial. Conversely, the proposed method reduces the maximum delay by a factor of $10^{3}$ when the traffic rate is high.

\subsection{Details of simulation methods in latency analysis}

In this subsection, we describe details of the numerical study shown in Sec. 3.1. To simulate shortdistance, small-scale communication networks such as data centers and in-vehicle networks, the following settings were considered:

- The bus length is equivalent to $L=100$ words.

- The message or packet size is $M=105$ words (equivalent to the interval between consecutive APs for the time synchronization method. For CSMA/CD, it is equivalent to 106 words because the last word of transmission is included.)

- The number of nodes is $N=10$ and they are randomly placed.

- $R$ denotes the traffic rate ranging from 0 to 1 . We assume that packets are arriving at the transmission queue of each node with an exponential distribution. Namely, packets arrive independently at nodes with a constant average interval. Let the average interval between packets at each node be given by $T_{\mathrm{PI}}$. The traffic rate in this study is defined by

$$
R=\frac{N M}{T_{\mathrm{PI}}},
$$

or equivalently $1 / T_{\mathrm{PI}}=R /(N M)$, which gives the rate parameter of the exponential distribution. With this definition, the smallest $T_{\mathrm{PI}}$ is given by $N M$ when $R=1$. In the numerical demonstration in Sec. 3.1, the traffic rate ranges from 0.01 to 0.99 .

- The simulation time is equivalent to $10^{7}$ words.

The details of the CSMA/CD-based arbitration method are as follows. 
1. When a node receives the header of a message addressed to itself:

(a) The node receives data during the length of the message size.

(b) When the last word of transmission is received after the message size, the received message is treated as a valid message.

2. When there is a message in the transmission queue, and the node is not waiting:

(a) The node starts sending the message.

(b) The node receives the downstream data while transmitting data, and check whether it is same as the transmitted message.

(c) If the transmitted message is different from the received downstream data, it means that a collision is induced. A jam signal is sent to notify the other nodes that a collision has occurred.

(d) Each node counts the number of collisions, denoted by $c$. After a collision, the node waits for durations specified randomly smaller than $2^{\min (10, c)}$ messages.

\section{Experimental demonstration}

\subsection{Information transfer}

The proposed method is based on precision time synchronization in data transmission. We experimentally evaluated the skew of information transmission using Wi-Wi to examine the validity of one of the most fundamental aspects of the proposed arbitration method. One remark here is that we utilize electrical cables instead of optical ones. This is because the examination herein addresses the first proof-of-concept experiment based on $\mathrm{Wi}$-Wi for data transmission between two nodes, whereas truly fast optical data transmission is not the primary goal at this stage.

Figure 5(a) shows a schematic of the experimental setup, and Fig. 5(b) shows a snapshot of the experimental apparatus where two nodes, i.e., node A and B, are interconnected while their timings are synchronized via Wi-Wi. Each node sends messages to the other node and to itself via the same route. A node starts sending a message when it is triggered by the edge of the pulse generated by Wi-Wi with a rate of 1 pulse per second (pps). The 1-pps trigger signals on both the nodes are synchronized after Wi-Wi is switched on. The messages are transmitted at the rate of $20 \mathrm{Mbps}$, which corresponds to the base clock signals $(10 \mathrm{MHz})$ supplied by Wi-Wi. This indicates that the time duration of a single bit corresponds to $50 \mathrm{~ns}$. The details of the experimental setup are described in Sec. 4.2.

We measured the skew between the first signals when Wi-Wi was activated and deactivated. In each case, we measured the skew nearly every $6 \mathrm{~s}$ to examine its time variation. Here the skew is given by the time difference defined by the arrival time of the message from node $\mathrm{B}$ minus the arrival time of the message from node A. As shown in Fig. 5(c), by activating Wi-Wi, the skew between the first signals can be reduced to nearly $1 / 100$ with respect to its value in the case where Wi-Wi is deactivated. On the contrary, even when the time synchronization is activated, a skew of at most 6 bits or $300 \mathrm{~ns}$ is observed. However, Fig. 6(b) shows that the skew can be maintained within \pm 1 bit of its initial value for about 10 min by activating Wi-Wi, but it increases by 300 bit when Wi-Wi is deactivated, as shown in Fig. 6(a). This implies that the skew can be controlled within 1 bit by offset adjustment, which is necessary for the proposed arbitration protocol to recognize the timing of message transmission.

Meanwhile, network time protocol (NTP) is a well-known and widely utilized protocol to synchronize the clocks of computers over a network, indicating that NTP could provide the same role that $\mathrm{Wi}$-Wi is offering. Therefore, for comparison, we measured the skew between the nodes when their timing information was calibrated by connecting them to an NTP server prior to the measurements. The details of the NTP used in the present study are described in Sec. 4.2.

Specifically, the connection to the NTP server was made before the measurement, and each node sent a message every second based on its own internal reference clock. Figures 6(c) and 6(d) show 
(a)

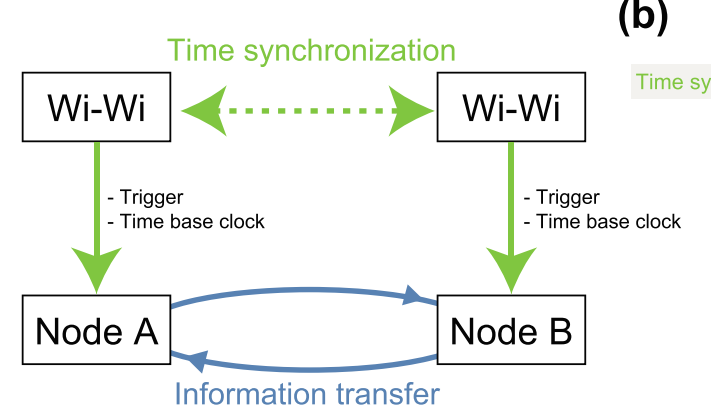

(c)

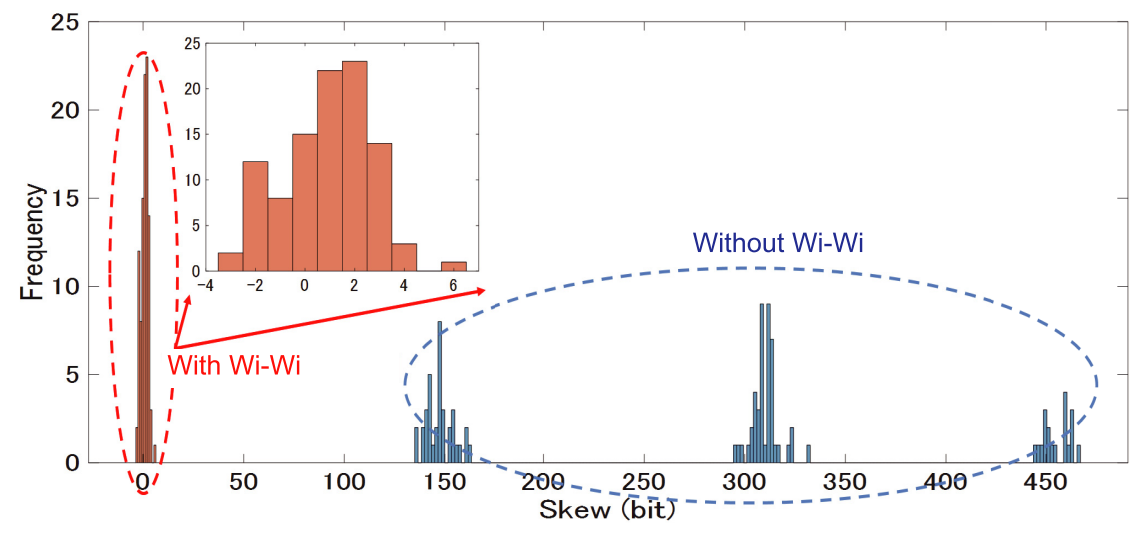

Fig. 5. Experimental demonstrations. (a) Schematic of the experimental setup. (b) Overview of the experimental setup. Each interface board is connected to the input/output $(\mathrm{I} / \mathrm{O})$ devices in the corresponding $\mathrm{PC}$ via the upper black wire. (c) Skew between the first signals when Wi-Wi is activated and deactivated. We conducted 100 measurements for each case.

the time evolutions of skew in two independent measurements. Note that the unit of skew is ms, not bit. First, if we focus on the skew at the beginning of the measurement, it is clear that the skew is in the ms range in both Figs. 6(c) and 6(d), even immediately after the connections to the NTP server. This skew is 5-6 orders of magnitude larger than that in the case based on Wi-Wi. More specifically, the initial skews observed in Figs. 6(a) and 6(d) are about $134 \mathrm{~ms}$ and $-10 \mathrm{~ms}$, respectively, which stems from the limitation of NTP stability. In addition, the slope of the graph is equal in these two figures, and the skew is reduced by approximately $2.5 \mathrm{~ms}$ per $10 \mathrm{~min}$. This indicates that the individual device-dependent variations, such as frequency differences between the reference clocks inside the two PCs and/or digital I/O devices, lead to timing variations. From this particular experiment, we observe that the reference clock on node B is faster than that on node A. These results indicate that not only the accuracy of the time synchronization by NTP is insufficient, but also that the time synchronization is easily lost due to the irregularity of of the reference clock between nodes.

\subsection{Experimental scheme for skew evaluation}

Here we describe technical details regarding the experimental demonstration shown in Sec. 4.1. First, Wi-Wi modules use 920-MHz wireless signal for time synchronization. The digital I/O devices used in the experiment were PCIe-6537B manufactured by National Instruments. The digital I/O devices were connected to interface board CB-2162 via the cable 195949A-01_C68-C68-D4. The digital I/O devices were controlled by LabVIEW 2019. The length of the wires used to connect the interface boards was $20 \mathrm{~cm}$, and the length of the wires between Wi-Wi and the interface boards was approximately $53 \mathrm{~cm}$. A message included 37 digits of 0 and 1 data. The computers used in the experiment were standard desktop machines: node A: Dell Precision 5820 (CPU: Xeon W-2102, $2.90 \mathrm{GHz}$, OS: Windows 10) and node B: Dell Precision 3620 (CPU: Core i5-6500, 3.20 GHz, OS: Windows 10). The NTP server was provided by the National Institute of Information and Communications Technology (ntp.nict.jp). This server is located in Nukui-Kitamachi, Koganei, Tokyo, Japan. The connection with the NTP 
(a)

Without Wi-Wi

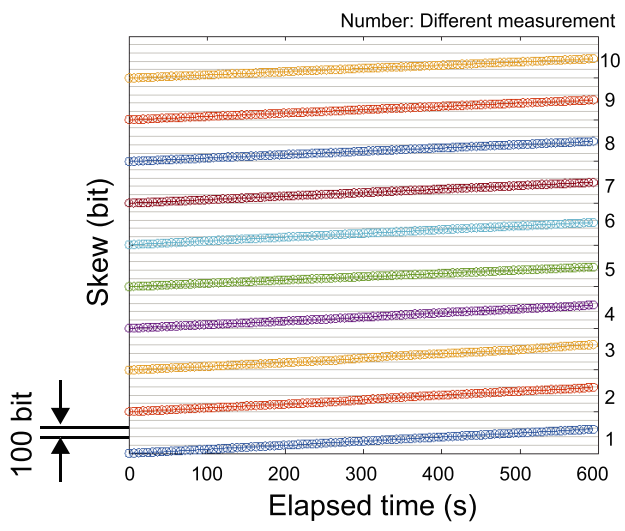

(c)

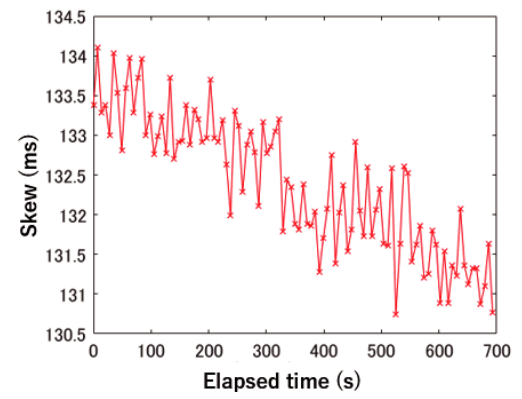

(b)

With Wi-Wi

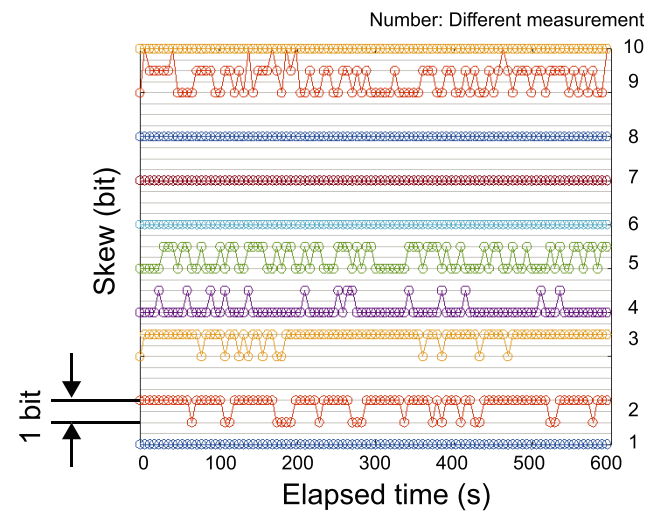

(d)

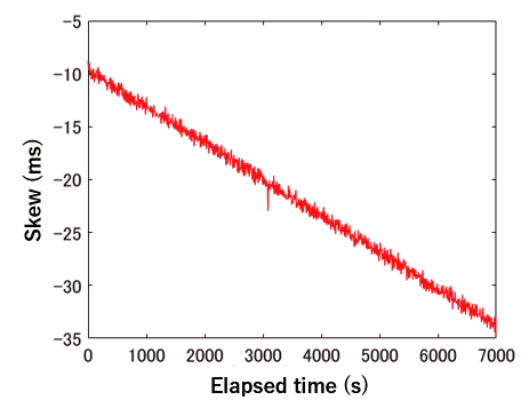

Fig. 6. Stability and instability of skew with and without Wi-Wi. (a) Ten measurements for the time variation of skew when $\mathrm{Wi}-\mathrm{Wi}$ is deactivated. The interval of grid lines is equivalent to 100 bits. (b) Ten measurements for the time variation of skew when $\mathrm{Wi}-\mathrm{Wi}$ is activated. Two intervals of grid lines are equivalent to 1 bit. (c,d) Time variation of skew when the nodes are connected to the NTP server before measurements. We performed (c) 100 and (d) 1000 measurements every $7 \mathrm{~s}$. In (d), 13 points are omitted due to data loss.

server was achieved using Windows built-in time setting.

\subsection{Future scope}

Before conclusion, we put a few remark regarding important research topics in the future. First, in the present study, we have assumed a ring-structure network due to its simplicity and high technological relevance to the optical ring network studied in the existing literature $[8,9]$. However, other network structures such as tree, star, and their combinations may be interesting for future investigations.

Second, in the experiment, we utilized an electrically-connected two-node system for the proofof-principle demonstrations. The transition to an all-optical and high-speed data line is one among several important technological upgrades. The experimental findings reported herein were based on the composition of the non-optical experimental apparatus described in Sec. 4.2; we have to reconfirm the associated properties when the data rate significantly increases in the future. However, we consider that the basic experimental results demonstrated in this paper will be just as valid when the data link is replaced with an optical one. The initial skew distribution shown in Fig. 5(c) might largely depend on the performance of Wi-Wi modules themselves rather than the technological details of data transfer. The time evolution of skew shown in Fig. 6 demonstrated the long-term stability of the system, which might be inherited in fast data rate systems. Meanwhile, we have to admit that the performances in the shorter time regime must be confirmed in the future when the technologies are upgraded.

Third, which is related to the second remark above, the packet rate of information transmission in the experiment is defined through the trigger signal supplied by the Wi-Wi module, which is 1 pps. However, this rate can be much higher in practical applications. In the future, we hope to address this issue using technological solutions including the latest field-programmable gate-array (FPGA) 
technology. The composition of such electrical circuits, Wi-Wi systems, and optical medium should be examined in the future.

\section{Conclusions}

We demonstrated the reducing arbitration overhead and ensuring information-transfer latency to realize the full potential of high-bandwidth optical communications and to facilitate data transfer applications in which real-time operation or short latency is critical. We proposed an arbitration protocol in a ring-structure network based on precision time synchronization via Wi-Wi, which ensured that the latency of data transfer was strictly bounded by an upper limit, while conventional CSMA/CD-based approach resulted in a very high latency especially in the cases with high traffic rate. Furthermore, we conducted a proof-of-principle experiment for data transfer between two electrically connected nodes that were time synchronized via Wi-Wi. We confirmed that the skew was not more than $300 \mathrm{~ns}$ and remained stable over time. Conversely, huge and unstable skew was observed in the absence of time synchronization. These results indicate that precise time synchronization is a vital resource to significantly reduce the communication overheads and to ensure that the latency is smaller than the pre-defined value for future networks and real-time applications.

\section{Acknowledgments}

This work was supported in part by the CREST project (JPMJCR17N2) funded by the Japan Science and Technology Agency and Grants-in-Aid for Scientific Research (JP17H01277, JP20H00233) funded by the Japan Society for the Promotion of Science.

\section{References}

[1] K. Bergman, J. Shalf, and T. Hausken, "Optical interconnects and extreme computing," Optics and Photonics News, vol. 27, pp. 32-39, April 2016.

[2] W. Zeng, M.A.S. Khalid, and S. Chowdhury, "In-vehicle networks outlook: Achievements and challenges," IEEE Communications Surveys \& Tutorials, vol. 18, no. 3, pp. 1552-1571, January 2016.

[3] N. Shiga, K. Kido, S. Yasuda, B. Panta, Y. Hanado, S. Kawamura, H. Hanado, K. Takizawa, and M. Inoue, "Demonstration of wireless two-way interferometry (Wi-Wi)," IEICE Communication Express, vol. 6, no. 2, pp. 77-82, November 2016.

[4] S. Yasuda, R. Ichikawa, Y. Hanado, S. Kawamura, H. Hanado, H. Iwai, K. Namba, Y. Okamoto, K. Fukunaga, T. Iguchi, and N. Shiga, "Horizontal atmospheric delay measurement using wireless two-way interferometry (Wi-Wi)," Radio Science, vol. 54, no. 7, pp. 572-579, June 2019.

[5] B.R. Panta, K. Kido, S. Yasuda, Y. Hanado, S. Kawamura, H. Hanado, K. Takizawa, M. Inoue, and N. Shiga, "Distance variation monitoring with wireless two-way interferometry (Wi-Wi)," Sensors and Materials, vol. 31, no. 7, pp. 2313-2321, July 2019.

[6] D.W. Hanson, "Fundamentals of two-way time transfers by satellite," Proceedings of the 43rd Annual Symposium on Frequency Control, pp. 174-178, May 1989.

[7] M. Fujieda, T. Gotoh, F. Nakagawa, R. Tabuchi, M. Aida, and J. Amagai, "Carrier-phase-based two-way satellite time and frequency transfer," IEEE Transactions on Ultrasonics, Ferroelectrics, and Frequency Control, vol. 59, no. 12, pp. 2625-2630, December 2012.

[8] M. Keri, E. Oki, and M. Matsuura, "Wavelength assignment in multi-carrier distributed optical ring networks with wavelength reuse," IEEE/OSA Journal of Optical Communications and Networking, vol. 3, no. 4, pp. 281-289, April 2011.

[9] P. Grani and S. Bartolini, "Design options for optical ring interconnect in future client devices," ACM Journal on Emerging Technologies in Computing Systems, vol. 10, no. 4, article 30, pp. 125, May 2014. 